\title{
Septic Vs colonizing group b streptococci differentially regulate inflammation and apoptosis in human coronary artery endothelial cells - a pilot study
}

\begin{abstract}
In this pilot study, we exemplify differences between a septic and a colonizing GBS strain during their interaction with Endothelial Cells by evaluating cytokine levels, surface and apoptosis-related molecules. These preliminary results indicate that in vitro infection using an exemplary septic GBS strain results in diminished activation of the innate immune response.
\end{abstract}

Keywords: streptococci, apoptosis, inflammation, endothelial cells, innate immunity, early onset sepsis

\author{
Volume 2 Issue 2 - 2015 \\ Claudia Loeffler, ${ }^{1,2}$ Juergen Loeffler, ${ }^{2}$ \\ Anna Kobsar,' Christian P Speer, ${ }^{3}$ Martin \\ Eigenthaler' \\ 'Institute of Clinical Biochemistry and Pathobiochemistry with \\ Division of Laboratory Medicine, University of Wuerzburg, \\ Germany \\ ${ }^{2}$ Department of Internal Medicine II, University of Wuerzburg, \\ Germany \\ ${ }^{3}$ University Children's Hospital, Germany
}

Correspondence: Claudia Loffler, Department of Internal Medicine II, University of Wuerzburg, D-97080 Wuerzburg, Germany, Tel +49 93। 20136404, Fax +49 93। 2016036405, Email Loeffler_c@ukw.de

Received: November 16,2014 | Published: April 18, 2015

Abbreviations: ATCC, american type culture collection; Casp, caspase; GBS, group B streptococcus; GS, colonizing GBS strain (clinical isolate); HCAEC, human coronar artery endothelial cells; IL-6, interleukin-6; IL-8, granulocyte chemotactic protein-1; LB, lysogeny broth; MCP-1, monocyte chemoattractant protein-1; PKB/ Akt, protein kinase B; VCAM-1, vascular cell adhesion molecule.

\section{Introduction}

Sepsis due to infections with Group B Streptococcus (GBS) is a common cause of morbidity and mortality in neonates and immune suppressed patients in Western countries. Two thirds of newborns are colonized post-partum and $0.2 \%$ suffer from pneumonia, meningitis and septicemia due to GBS infection. ${ }^{1,2}$ Fifty percent of surviving children are seriously affected by late effects, including blindness, deafness and epilepsy. ${ }^{3}$ GBS grow invasively by penetrating cellular barriers utilizing characteristic virulence factors ${ }^{4,5}$ basically exploiting endothelial dysfunction. ${ }^{6}$ It is a pivotal question why some patients develop severe sepsis, whereas another collective is simply acting as carriers. Therefore we focused on potentially relevant key mechanisms in the pathophysiology of GBS infection. This includes cell apoptosis, cytokine response and inflammation.

\section{Materials and methods}

By way of example, a septic reference strain of Streptococcus agalactiae Lancefield's group B (ATCC ${ }^{\circledR} 13813$ strain Lehmann and Neumann, serotype V, non-hemolytic, clinical symptoms sepsis, LGC Promochem, Wesel, Germany) and a colonizing GBS strain (GS130903, isolated from ear swab, University Children's Hospital, non-typable serotype, clinical symptoms none) were selected for in vitro culturing with Primary Human Coronary Artery Endothelial Cells (HCAEC). Culturing assays for bacteria and HCAEC were performed as described before. For ELISA experiments (MCP-1, IL6, IL-8, R\&D Systems, Minneapolis/USA), cell culture supernatants and lysates were collected after 4, 8 and 24hours, respectively and analyzed as previously described. For simultaneous Western Blot analysis (Cl. Casp 3: 1:1000, milk, rabbit; Casp 8: 1:1000, BSA, mouse; PKB/Akt: 1:1000, BSA, rabbit; Cell Signaling), protein extracts were prepared, electro-transferred and blocked as described before ${ }^{7}$. Loading control was performed with $\beta$-actin. For measuring surface molecules by Flow cytometry E-Selectin (anti-CD62E), VCAM-1 (anti-CD106) cells were incubated with primary and secondary antibodies (FITC-conjugated, 1:20, mouse, Chemicon Upstate) and afterwards fixed with $4 \%$ Formaldehyde. In preliminary experiments we could not detect any change after 4 and 8hours of infection. Therefore incubation time was elongated to 24,48 and 72 hours.

Data represent means $+/$ - standard errors of the means (SEM). Differences between groups were tested using Student's t-test. Differences were considered to be significant at $\mathrm{p}<0.05$. Biological replicates were performed at least three to five times.

\section{Results}

In the present study, we sequentially analyzed the effects of septic and colonizing GBS on HCAEC and could demonstrate by way of example.

\section{Differential secretion of pro-inflammatory cytokines and chemokines}

After infection with the colonizing GBS strain, MCP-1 secretion to culture medium was markedly augmented $(5.3$-fold; $p<0.01)$ after 24hours of infection compared to un-stimulated cells (defined as $100 \%$ ), whereas the septic strain ATCC ${ }^{13813}$ led to lower MCP-1 levels (2.4-fold; $\mathrm{p}=0.03$ ) after 24hours.

In contrast, IL-6 levels were higher, when cells were exposed to the septic GBS strain (10.7-fold; $\mathrm{p}=0.01$ ), compared to the colonizing 
strain (5.3-fold; $p<0.01)$. Both strains caused nearly identical increases of IL-8 levels (3.2-fold $(\mathrm{p}<0.01)$ and 3.4-fold ( $\mathrm{p}=0.01)$, respectively) (Figure 1).

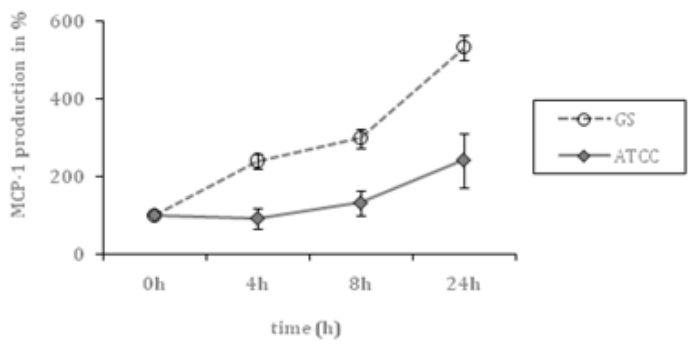

Figure IA Time course of MCP-I secretion by HCAEC, co-incubated with a septic (ATCC) and colonizing (GS) GBS strain, respectively (cell culture supernatants).

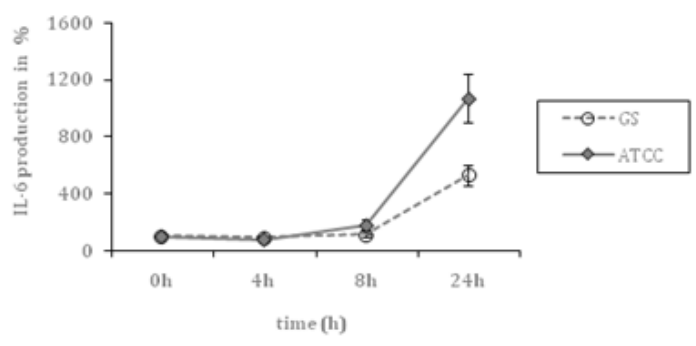

Figure IB Time course of IL- 6 secretion by HCAEC, co-incubated with septic (ATCC) and colonizing GBS (GS) strain, respectively (cell culture supernatants)

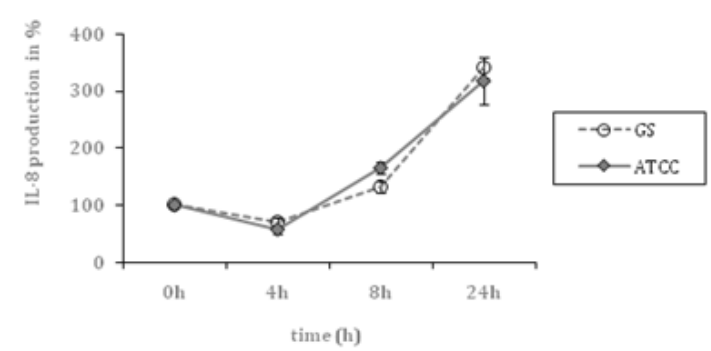

Figure IC Time course of IL-8 secretion by HCAEC, co-incubated with septic (ATCC) and colonizing GBS (GS) strain, respectively (cell culture supernatants).

Figure I Differential secretion of pro-inflammatory cytokines and chemokines Data shown represent mean levels in percent \pm SEM of at least 4 independent experiments. The un-stimulated control is defined as 100 percent. *indicates statistical significant difference $(p<0.05)$.

\section{Differential regulation of apoptotic and anti-apoptotic signaling-pathways}

Cleavage of Caspase 3 was substantially increased after infection with the septic strain (maximum 52-fold; $\mathrm{p}=0.04$ ) compared to the colonizing strain (maximum 31-fold; $\mathrm{p}=0.04$ ) after 24hours of incubation.
Both strains led to similar activation of Caspase 8 (7-fold and 9-fold, respectively; $\mathrm{p}=0.05$ and 0.04 ). Interestingly, we revealed substantially differential inhibition of the $\mathrm{PKB} / \mathrm{Akt}$ signaling pathway (septic strain: 15 -fold, $\mathrm{p}=0.04$ vs. colonizing strain: 155 -fold, $\mathrm{p}=0.01$ ) after $24 \mathrm{~h}$ of infection (Figure 2).

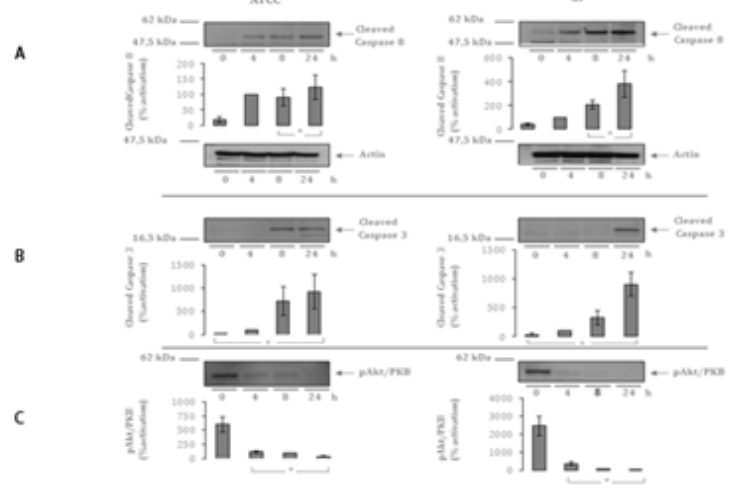

Figure $2 \mathrm{~N}=57$; Epidemiological distribution of the pathological fractures, traumatic fractures, and nonunion.

Figure 2A Autoradiographs and quantitative analysis of Western Blot Assays demonstrating up-regulation of Cleaved Caspase 8 after 4, 8 and 24 $\mathrm{h}$ in comparison to un-stimulated HCAEC(cell culture lysates). Exemplary presentation of loading controls with B-actin.

Figure 2B Autoradiographs and quantitative analysis of Western Blot Assays demonstrating up-regulation of Cleaved Caspase 3 after 4, 8 and 24 h in comparison to un-stimulated HCAEC (cell culture lysates).

Figure 2C Autoradiographs and quantitative analysis of Western Blot Assays demonstrating up-regulation of PKB/Akt after 4, 8 and $24 \mathrm{~h}$ in comparison to un-stimulated HCAEC (cell culture lysates).

Data shown represent mean expression in percent \pm the standard error of the mean (SEM) of 4 independent experiments. The results after 4 hours are defined as 100 percent. * indicates statistical significant difference $(p<0.05)$.

\section{Differential activation of surface molecules}

Only the colonizing strain GS130903 led to VCAM-1 upregulation only after 72 hours $(1.5$-fold; $\mathrm{p}=0.03)$. After 48 hours of stimulation with both strains, we found nearly comparable mean fluorescence values of E-Selectin surface molecules (1.5 and 2-fold respectively; $\mathrm{p}=0.01$ and $<0.01$ ). Interestingly, E-Selectin expression was diminished after 72hours of co-cultivation of HCAEC with the colonizing strain (1.4-fold), whereas we could not show any marked difference after infection with the septic strain ATCC ${ }^{1} 13813$.

\section{Discussion}

Our results indicate that both strains specifically induce the secretion of defined pro-inflammatory cytokines and chemokines. Production of IL-6 was markedly augmented, especially after infection with the septic GBS strain, whereas MCP-1 levels were significantly higher after stimulation with the colonizing strain. Levels of IL-8 were nearly identical following stimulation with both strains. These cytokines primarily activate monocytes and induce their migration to sides of infection. Neutrophils and monocytes are part of the innate immune system, which provides a first line of defense against bacterial infection, thereby playing a crucial role concerning defense mechanisms of the neonate. ${ }^{8}$ Especially in preterm infants monocytes may have an intrinsic deficiency to recognize and to respond to GBS. ${ }^{9}$ One reason might be an impaired function of Toll-like receptors. ${ }^{10-12}$

These results demonstrate that endothelial cells contribute in a relevant manner to the innate immune response during GBS infection. 
This is in accordance with other macro-vascular cell types, such as human umbilical vein-derived endothelial cells ${ }^{5}$ and with other infectious agents like LPS. ${ }^{13}$ Lower MCP-1 levels after infection with the septic reference strain might lead to insufficient recognition by the innate immune system. Furthermore, significant higher IL6- levels after infection with the septic GBS strain could explain activation of pro-thrombotic processes via induction of tissue damage, ${ }^{14}$ stimulation of megakaryocytes and platelet activation ${ }^{15}$ during an acute phase reaction. Similar results have been published by Berner et al., ${ }^{16}$ who found significantly higher IL-6 levels in cord blood mononuclear cells after stimulation with neonatal sepsis compared to colonizing GBS strains. ${ }^{16}$

To differentiate between cytokine release and modified cytokine production, we also performed ELISA from cell culture lysates (data not shown). The production of IL-6 was increased in a time dependent manner, which was nearly identical with IL-6 levels in supernatants. In contrast, production of IL-8 and MCP-1 differed in-between the sources. We found decreased levels of IL- 8 and MCP-1 after infection with the septic reference strain and hypothesized that this fact might also be at least partly due to an insufficient innate immune response. Further analyses should be carried out focusing MCP-1 and IL-8 levels in cell culture lysates after infection with colonizing strains (Table 1).

Table I Differential expression of selected markers

\begin{tabular}{|c|c|c|}
\hline & $\begin{array}{l}\text { Colonizing } \\
\text { Reference } \\
\text { Strain }\end{array}$ & $\begin{array}{l}\text { Septic } \\
\text { Reference } \\
\text { Strain }\end{array}$ \\
\hline VCAM-I & $\uparrow$ & $\leftrightarrow$ \\
\hline $\begin{array}{l}\text { Maximal mean Fluorescence Increase } \\
\text { [72 hours] }\end{array}$ & I.5-fold & No change \\
\hline p-value & 0.03 & 0.2 \\
\hline E-Selectin & $\uparrow$ & $\uparrow$ \\
\hline $\begin{array}{l}\text { Maximal Mean Fluorescence Increase } \\
\text { [48 hours] }\end{array}$ & 2-fold & I.5-fold \\
\hline p-value & $<0.01$ & 0.01 \\
\hline Cl. Caspase 3 & $\uparrow$ & $\uparrow \uparrow$ \\
\hline $\begin{array}{l}\text { Maximum of Activation } \\
\text { [24 hours] }\end{array}$ & 31 -fold & 52 -fold \\
\hline p-value & 0.04 & 0.04 \\
\hline Caspase 8 & $\uparrow$ & $\uparrow$ \\
\hline $\begin{array}{l}\text { Maximum of Activation } \\
\text { [24 hours] }\end{array}$ & 9-fold & 7-fold \\
\hline p-value & 0.04 & 0.05 \\
\hline $\mathrm{PKB} / \mathrm{Akt}$ & $\downarrow \downarrow \downarrow$ & $\downarrow$ \\
\hline $\begin{array}{l}\text { Maximum of Inhibition } \\
\text { [24 hours] }\end{array}$ & I55-fold & I5-fold \\
\hline p-value & 0.01 & 0.04 \\
\hline MCP-I & $\uparrow \uparrow$ & $\uparrow$ \\
\hline Maximal Mean Production in \%; [24 hours] & 532 & 241 \\
\hline p-value & $<0.01$ & 0.03 \\
\hline IL-6 & $\uparrow$ & $\uparrow \uparrow$ \\
\hline Maximal Mean Production in \%; [24 hours] & 531 & 1067 \\
\hline p-value & $<0.01$ & 0.01 \\
\hline IL-8 & $\uparrow$ & $\uparrow$ \\
\hline Maximal Mean Production in \%; [24 hours] & 340 & 318 \\
\hline $\mathrm{p}$-value & $<0.01$ & 0.01 \\
\hline
\end{tabular}

Regulation after infection with a GBS septic (ATCC®I38I3) in comparison with a colonizing (GSI30309) GBS strain

VCAM-I:Vascular Cell Adhesion Molecule I; PKB/Akt: Protein Kinase B; MCP I:Monocyte Chemo attractant Protein- I; IL-6: Interleukin-6; IL-8: Interleukin-8
In addition, we could demonstrate that cleavage of Caspase 3 and Caspase 8 was increased (Figure 2). Induction of apoptosis by GBS could be previously demonstrated for macrophages, monocytes and fibroblasts, but not for other cell lines, which in consequences implicates a cell specific phenomenon. ${ }^{17}$ It is remarkable that the septic GBS strain showed a stronger activation of Caspase 3, compared to the colonizing strain. We assume that this stronger Caspase 3 activation is, among other factors related to the presence of $\beta$-hemolysin, as previously demonstrated for macrophage ${ }^{18}$ and monocyte apoptosis. ${ }^{19}$ GBS-induced apoptosis is inhibited by the Caspase-3 inhibitor DEVDCHO. ${ }^{17}$ In consequence, induction of Caspase- 3 might be responsible for tissue damage, leading thereby to dysregulation of hemostasis. In contrast, the colonizing strain causes a stronger inhibition of the $\mathrm{PKB} /$ Akt survival pathway, which could suppress the dissemination of the infection. Levels of Caspase 8 cleavage were comparable.

Finally, we revealed differential regulation of endothelial surface molecules. Several groups could previously show unequivocal expression of adhesion molecules after cytokine stimulation, ${ }^{20}$ which might be also a possible mechanism for microbe induced regulation. The expression of VCAM-1 was markedly augmented after infection with the colonizing strain compared to the septic strain, thereby facilitating monocyte migration and activation of the innate immune system.

\section{Conclusion}

In summary, exposure of neonates to GBS represents a strong challenge to the local immunity. Neonates acquire GBS at birth when the neonatal lung may be infected with a substantial inoculum from amniotic fluid. Although, GBS contributes to a poor inflammatory response in the neonatal lung, fast and potent activation of the innate immune system by macrophages, monocytes, pulmonary endothelia and epithelia but also by cells of the human vessels is mandatory. Surprisingly, coronary artery endothelial cells seem to play a substantial role in the innate immune response against GBS as well. Future projects need to focus on functional analyses of key players involved in innate immunity and enlarge the number of GBS strains.

\section{Acknowledgments}

This study was supported by a grant of the "Interdisziplinäres Zentrum für Klinische Forschung", project A33 and funded by the German Research Foundation (DFG) and the University of Wuerzburg in the funding programme Open Access Publishing.

\section{Conflicts of interest}

Author declares there are no conflicts of interest.

\section{Funding}

None.

\section{References}

1. Larsen JW, Sever JL. Group B Streptococcus and pregnancy: a review. Am J Obstet Gynecol. 2008;198(4):440-450.

2. Baker CJ. The spectrum of perinatal group B streptococcal disease. Vaccine. 2013;31(Suppl 4):D3-D6.

3. McKenna DS, Iams JD. Group B streptococcal infections. Seminars in perinatology. 1998;22(4):267-276

4. Schwarz-Linek U, Hook M, Potts JR. The molecular basis of fibronectin-mediated bacterial adherence to host cells. Mol Microbiol. 2004;52(3):631-641. 
5. Doran KS, Liu GY, Nizet V. Group B streptococcal beta-hemolysin/ cytolysin activates neutrophil signaling pathways in brain endothelium and contributes to development of meningitis. $J$ Clin Invest 2003;112(5):736-744

6. Bannerman DD, Goldblum SE. Direct effects of endotoxin on the endothelium: barrier function and injury. Lab Invest 1999;79(10):1181-1199.

7. Beyrich C, Loffler J, Kobsar A, et al. Infection of human coronary artery endothelial cells by group B streptococcus contributes to dysregulation of apoptosis, hemostasis, and innate immune responses. Mediators Inflamm. 2011;2011:971502.

8. Fleer A, Krediet TG. Innate immunity: toll-like receptors and some more. A brief history, basic organization and relevance for the human newborn. Neonatology. 2007;92(3):145-157.

9. Dembinski J, Behrendt D, Martini R, et al. Modulation of pro- and antiinflammatory cytokine production in very preterm infants. Cytokine. 2003;21(4):200-206.

10. Sadeghi K, Berger A, Langgartner M, et al. Immaturity of infection control in preterm and term newborns is associated with impaired tolllike receptor signaling. J Infect Dis. 2007;195(2):296-302.

11. Andrade EB, Alves J, Madureira P, et al. TLR2-Induced IL-10 Production Impairs Neutrophil Recruitment to Infected Tissues during Neonatal Bacterial Sepsis. J Immunol . 2013;191(9):4759-4768.

12. Glaser K, Speer CP. Toll-like receptor signaling in neonatal sepsis and inflammation: a matter of orchestration and conditioning. Expert Rev Clin Immunol. 2013;9(12):1239-1252.
13. Jehle AB, Li Y, Stechschulte AC, et al. Endotoxin and mast cell granule proteases synergistically activate human coronary artery endothelial cells to generate interleukin-6 and interleukin-8. J Interferon Cytokine Res. 2000;20(4):361-368.

14. Westlin WF, Gimbrone MA Jr. Neutrophil-mediated damage to human vascular endothelium. Role of cytokine activation. Am J Pathol. 1993;142(1):117-128.

15. Siauw C, Kobsar A, Dornieden C, et al. Group B streptococcus isolates from septic patients and healthy carriers differentially activate platelet signaling cascades. Thromb Haemost. 2006;95(5):836-849.

16. Berner R, Csorba J, Brandis M. Different cytokine expression in cord blood mononuclear cells after stimulation with neonatal sepsis or colonizing strains of Streptococcus agalactiae. Pediatr Res. 2001;49(5):691-697.

17. Fettucciari K, Rosati E, Scaringi L, et al. Group B Streptococcus induces apoptosis in macrophages. J Immunol. 2000;165(7):3923-3933.

18. Liu GY, Doran KS, Lawrence T, et al. Sword and shield: linked group B streptococcal beta-hemolysin/cytolysin and carotenoid pigment function to subvert host phagocyte defense. Proc Natl Acad Sci US A. 2004;101(40):14491-14496.

19. Halaas O, Liabakk NB, Vik R, et al. Monocytes stimulated with group B streptococci or interferons release tumour necrosis factor-related apoptosis-inducing ligand. Scandinavian journal of immunology. 2004;60(1-2):74-81.

20. Constantin G, Piccio L, Bussini S, et al. Induction of adhesion molecules on human schwann cells by proinflammatory cytokines, an immunofluorescence study. J Neurol Sci. 1999;170(2):124-130. 